\title{
Resolving the stellar activity of the Mira AB binary with ALMA ${ }^{\star}$
}

\author{
W. H. T. Vlemmings ${ }^{1}$, S. Ramstedt ${ }^{2}$, E. O'Gorman ${ }^{1}$, E. M. L. Humphreys ${ }^{3}$, M. Wittkowski ${ }^{3}$, \\ A. Baudry ${ }^{4,5}$, and M. Karovska ${ }^{6}$ \\ ${ }^{1}$ Department of Earth and Space Sciences, Chalmers University of Technology, Onsala Space Observatory, 43992 Onsala, Sweden \\ e-mail: wouter.vlemmings@chalmers.se \\ 2 Department of Physics and Astronomy, Uppsala University, Box 516, 75120 Uppsala, Sweden \\ 3 ESO Karl-Schwarzschild-Str. 2, 85748 Garching, Germany \\ ${ }^{4}$ Université de Bordeaux 1, LAB, UMR 5804, 33270 Floirac, France \\ 5 CNRS, LAB, UMR 5804, 33270 Floirac, France \\ 6 Smithsonian Astrophysical Observatory, MS 70, 60 Garden Street, Cambridge, MA 02138, USA
}

Received 25 March 2015 / Accepted 18 April 2015

\section{ABSTRACT}

\begin{abstract}
Aims. We present the size, shape, and flux densities at millimeter continuum wavelengths, based on ALMA science verification observations in Band $3(\sim 94.6 \mathrm{GHz})$ and Band $6(\sim 228.7 \mathrm{GHz})$, from the binary Mira A (o Ceti) and Mira B.

Methods. The Mira AB system was observed with ALMA at a spatial resolution down to 25 mas. The extended atmosphere of Mira A and the wind around Mira B sources were resolved, and we derived the sizes of Mira A and of the ionized region around Mira B. The spectral indices within Band 3 (between 89-100 GHz) and between Bands 3 and 6 were also derived.

Results. The spectral index of Mira A is found to change from $1.71 \pm 0.05$ within Band 3 to $1.54 \pm 0.04$ between Bands 3 and 6 . The spectral index of Mira B is $1.3 \pm 0.2$ in Band 3, in good agreement with measurements at longer wavelengths; however, it rises to $1.72 \pm 0.11$ between the bands. For the first time, the extended atmosphere of a star is resolved at these frequencies, and for Mira A the diameter is $\sim 3.8 \times 3.2 \mathrm{AU}$ in Band 3 (with brightness temperature $T_{b} \sim 5300 \mathrm{~K}$ ) and $\sim 4.0 \times 3.6 \mathrm{AU}$ in Band $6\left(T_{b} \sim 2500 \mathrm{~K}\right)$. Additionally, a bright hotspot $\sim 0.4 \mathrm{AU}$, with $T_{b} \sim 10000 \mathrm{~K}$, is found on the stellar disk of Mira A. The size of the ionized region around the accretion disk of Mira B is found to be $\sim 2.4 \mathrm{AU}$.

Conclusions. The emission around Mira B is consistent with emission from a partially ionized wind of gravitationally bound material from Mira A close to the accretion disk of Mira B. The Mira A atmosphere does not fully match predictions with brightness temperatures in Band 3 significantly higher than expected, potentially owing to shock heating. The hotspot is very likely due to magnetic activity and could be related to the previously observed X-ray flare of Mira A.
\end{abstract}

Key words. stars: AGB and post-AGB - stars: atmospheres - binaries: close - stars: individual: Mira AB

\section{Introduction}

Mira is the closest symbiotic binary star (at $92 \mathrm{pc}$, van Leeuwen 2007), consisting of a regularly pulsating, mass-losing asymptotic giant branch (AGB) primary, Mira A (o Cet), and a companion, Mira B (VZ Cet), which is believed to be a white dwarf. Resolved UV observations of the two components were first published in Karovska et al. (1997), and they revealed material flowing from Mira A to Mira B.

A soft X-ray outburst was detected from Mira A in 2003 (Karovska et al. 2005). Since then, the system has been closely monitored at different wavelengths to better understand the cause and effects of the X-ray outburst. The $\mathrm{OH}$ maser emission is also varying and the most recently observed $\mathrm{OH}$ maser flare occurred in 2009 (Etoka et al. 2010). Although the OH flaring can be correlated with variations in the optical lightcurve and the UV line emission occuring on the same time scales, a consistent model that explains the transient phenomena detected by different probes is still missing.

The submm emission from the Mira $\mathrm{AB}$ system around the $\mathrm{CO}(J=3 \rightarrow 2)$ line at $345 \mathrm{GHz}$ was observed with ALMA in Cycle 1 (Ramstedt et al. 2014). The CO maps show that the

* Appendices are available in electronic form at http: //www . aanda.org circumstellar gas has been shaped by several different dynamical processes during the evolution of the star. In the continuum emission centered on $338 \mathrm{GHz}$, the binary pair is marginally resolved. In this paper, we present new ALMA continuum observations of the system taken as part of the ALMA long-baseline science verification campaign (ALMA partnership et al. 2015).

\section{Observations and analysis}

Mira was observed on 17 and 25 October 2014 (Band 3) and 29 October and 1 November 2014 (Band 6). Here we present results from the dual polarization continuum spectral windows. In Band 3, three continuum spectral windows were observed with 128 channels over a $2 \mathrm{GHz}$ bandwidth centered on 89.176 GHz, 91.234 GHz, and 99.192 GHz. In Band 6, one continuum $2 \mathrm{GHz}$ spectral window with 128 channels was centered on $228.558 \mathrm{GHz}$. We used the data products provided by the ALMA observatory except that the two epochs for each observing band were treated separately. Because the sources are strongly detected in each epoch, it allowings for study of structural and flux changes. Self-calibration was performed on the continuum spectral windows that were averaged from 128 to 8 channels across the $2 \mathrm{GHz}$ bandwidth. Flux density calibration was performed using a number of different quasars for the 

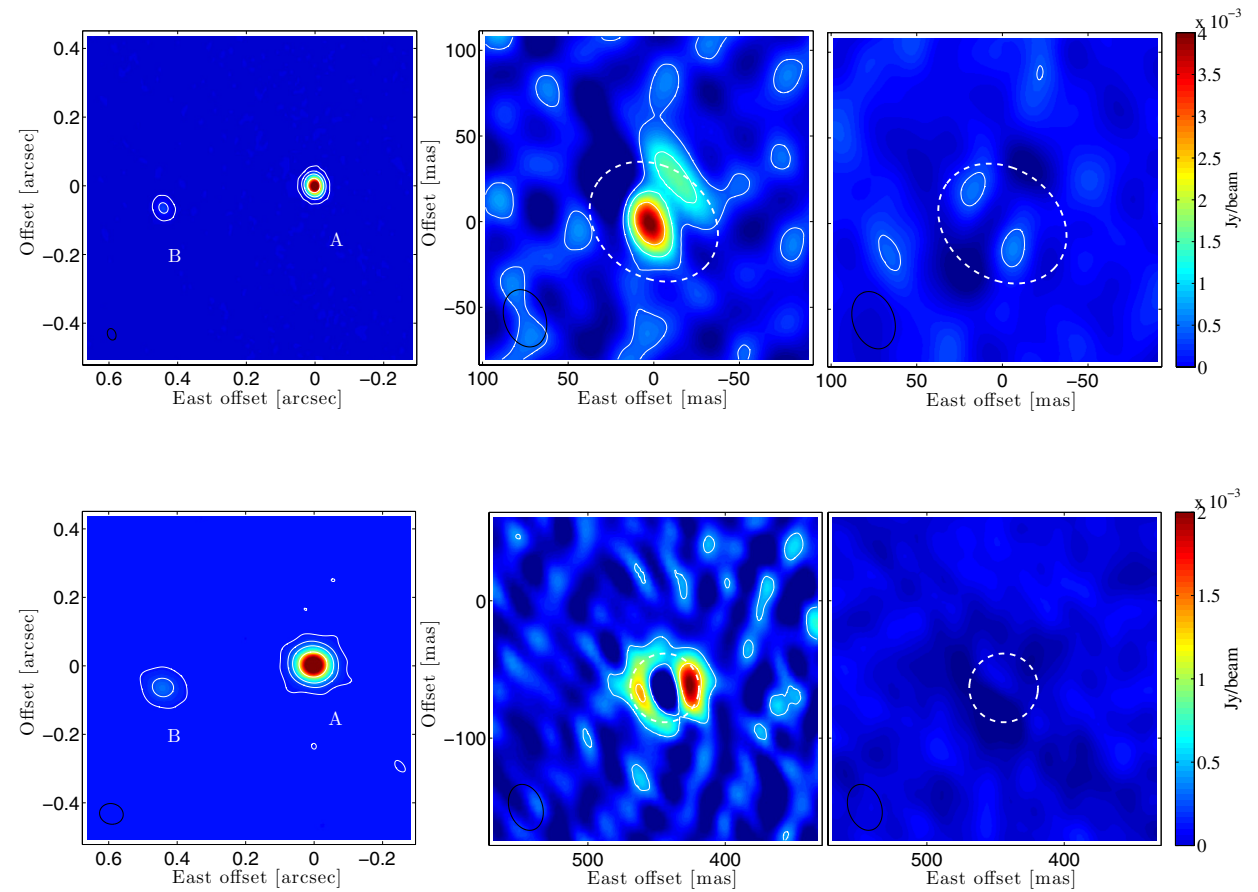

Fig. 1. Continuum image of the Mira $A B$ binary at Band 6 (left). Residuals in Band 6 at the location of Mira A after subtracting the best fit disc model (middle) and the disc model including a compact Gaussian hotspot (right) from the visibilities. The dashed ellipse indicates the size of the fitted stellar disc. Contours in all three plots are drawn at 3,30,90,270,810 $\sigma$ with $\sigma=$ $140 \mu \mathrm{Jy}$ beam $^{-1}$. The beam size is shown in black in the bottom left corner of all three figures.

Fig. 2. Continuum image of the Mira $A B$ binary at Band $3(l e f t)$. Residuals are shown for Band 6 at the location of Mira B after subtracting the best fit delta function (middle) and extended Gaussian (right). The dashed circle indicates the FWHM of the fitted Gaussian. Contours in all three plots are drawn at $3,30,90,270,810 \sigma$ with $\sigma=40 \mu \mathrm{Jy}$ beam $^{-1}$ in Band 3 and $\sigma=$ $140 \mu \mathrm{Jy}_{\text {beam }}^{-1}$ in Band 6 . The beam size is shown in the bottom left of all three figures.

Table 1. $u v$-fitting results.

\begin{tabular}{|c|c|c|c|c|c|c|c|}
\hline Epoch & $\begin{array}{c}v \\
{[\mathrm{GHz}]}\end{array}$ & Shape & $\begin{array}{c}S_{v} \\
{[\mathrm{mJy}]}\end{array}$ & $\begin{array}{c}\text { Major axis/fwhm } \\
{[\mathrm{mas}]}\end{array}$ & $\begin{array}{l}\text { Axis ratio } \\
\text { major/minor }\end{array}$ & $\begin{array}{c}\text { Position angle } \\
{\left[^{\circ}\right]} \\
\end{array}$ & Spectral index \\
\hline \multicolumn{8}{|l|}{ Mira A } \\
\hline 17 Oct. 2014 & 94.2 & Disc & $35.03 \pm 0.04$ & $41.8 \pm 0.4$ & $1.20 \pm 0.01$ & $54 \pm 2$ & $1.73 \pm 0.09$ \\
\hline 25 Oct. $2014^{a}$ & 94.2 & Disc & $34.52 \pm 0.04$ & $38.6 \pm 0.4$ & $1.28 \pm 0.01$ & $54 \pm 1$ & $1.70 \pm 0.04$ \\
\hline \multirow[t]{2}{*}{29 Oct. 2014} & 228.67 & Disc & $137.8 \pm 0.2$ & $43.28 \pm 0.07$ & $1.13 \pm 0.02$ & $51.0 \pm 0.5$ & \\
\hline & 228.67 & Gaussian & $10.13 \pm 0.07$ & $4.6 \pm 0.5$ & 1.0 & & \\
\hline \multirow[t]{2}{*}{01 Nov. 2014} & 228.67 & Disc & $140.0 \pm 0.2$ & $43.36 \pm 0.06$ & $1.12 \pm 0.02$ & $50.8 \pm 0.6$ & $\ldots$ \\
\hline & 228.67 & Gaussian & $8.98 \pm 0.07$ & $4.7 \pm 0.5$ & 1.0 & $x=$ & $\ldots$ \\
\hline \multicolumn{8}{|l|}{ Mira B } \\
\hline 17 Oct. 2014 & 89.06 & Gaussian & $2.50 \pm 0.04$ & $24 \pm 2$ & 1.0 & $\ldots$ & $1.3 \pm 0.2$ \\
\hline 25 Oct. $2014^{a}$ & 89.06 & Gaussian & $2.25 \pm 0.04$ & $18 \pm 2$ & 1.0 & $\ldots$ & $1.3 \pm 0.3$ \\
\hline 29 Oct. 2014 & 228.67 & Gaussian & $12.16 \pm 0.09$ & $25.7 \pm 0.3$ & 1.0 & $\ldots$ & $\ldots$ \\
\hline 01 Nov. 2014 & 228.67 & Gaussian & $11.98 \pm 0.09$ & $26.2 \pm 0.3$ & 1.0 & $\ldots$ & $\ldots$ \\
\hline
\end{tabular}

Notes. ${ }^{(a)}$ The size of both Mira A and B are likely underestimated at the second epoch due to a marginally extended flux calibrator J2258-2758.

two bands and epochs. The calibrators are listed in Appendix A. Based on the uncertainty of the catalog values due to quasar variability and the flux densities measured for the phase and bandpass calibrators in the different data sets, we conservatively estimate an absolute flux density calibration uncertainty of $\sim 5 \%$ in both observing bands.

Most of the data analysis was performed directly on the $u v$ data in order to avoid image deconvolution artifacts, but images were also created using the CASA task clean with Briggs 0.5 weighing of the visibilities. This resulted in a beam of $72 \times$ 64 mas and a position angle of $78.8^{\circ}$ on 17 October and $68 \times$ 57 mas at a position angle of $73.3^{\circ}$ on 25 October for Band 3. For Band 6 both days reached the same resolution of $34 \times$ 24 mas with a position angle of $22.4^{\circ}$ on 29 October and $16.0^{\circ}$ on 1 November. The rms sensitivity was $\sim 40 \mu \mathrm{Jy}$ beam $^{-1}$ in both epochs of the Band 3 and $\sim 130 \mu \mathrm{Jy} \mathrm{beam}^{-1}$ in both epochs of the Band 6 observations. The images in Band 3, with $>10 \%$ fractional bandwidth coverage, were made using the multi-frequency synthesis option in clean with two Taylor coefficients to model the frequency dependence. This allowed us to determine the spectral index at the $\sim 94.2 \mathrm{GHz}$ representative frequency.

To analyze the $u v$-data directly, we used the UVMULTIFIT code (Martí-Vidal et al. 2014). We find that the observations of October 25 in Band 3 are probably affected by a marginally resolved quasar flux calibrator, producing systematically smaller sizes, so we do not consider this epoch in our analysis.

\section{Results}

We present a representative image at the two frequencies in Fig. 1 (left, band 6) and Fig. 2 (left, band 3). The relative astrometry of the observations is discussed in Appendix B. To determine the size and shape of both Mira A and Mira B, we performed $u v$-fitting. For both sources we attempted fits to unresolved delta functions, to elliptical and circular Gaussians, and to elliptical and circular disks. The best fit parameters for Mira A and $\mathrm{B}$ at each epoch and observing band are presented in Table 1 . As indicated in Fig. 2 (right), the emission from Mira B is clearly extended, and the best fits were obtained using a circular 
Gaussian with a spectral index of $\alpha=1.3$. Within the fitted errors and estimated absolute flux density uncertainties, the flux density of Mira B is roughly constant at the two epochs in both bands. Between Bands 3 and 6, the spectral index is $1.72 \pm 0.11$, when taking all flux density uncertainties into account. Ignoring the observations of 25 October, the size is consistent between the different frequencies with a full-width half-maximum (FWHM) of $\approx 26$ mas, which corresponds to $\sim 2.4$ AU.

For Mira A, the best fits to the Band 3 data were produced with an elliptical disk with $\alpha \approx 1.7$. The flux density is nearly constant during both days at each observing frequency. The spectral index between Bands 3 and 6, however, is shallower with $\alpha=1.54 \pm 0.04$. The disk is clearly elongated with a major axis of 41.8 mas (3.8 AU), axis ratio of $\sim 1.2$, and position angle $\sim 54^{\circ}$. However, in Band 6, we found, as indicated in Fig. 1, a significant component remaining after subtracting the best fit disk model. This component, consistently at both epochs, could be fit by an additional compact Gaussian offset by $\sim 3$ mas from the disk center. Fitting both disk and Gaussian simultaneously signicantly reduced the residuals in the image. The disk is larger at Band 6 compared to Band 3, with an average size of 43.32 mas (3.99 AU) and an axis ratio of $\sim 1.1$.

\section{Discussion}

\subsection{Mira $A$}

\subsubsection{Size and shape}

Mira A has long been known to have an asymmetric stellar disk (e.g., Karovska et al. 1991; Haniff et al. 1992; Wilson et al. 1992; Quirrenbach et al. 1992). The position angle and axis ratio, however, has been shown to be quite variable across the stellar phase. Most observations taken around maximum phase are found to have a position angle of the major axis between $\sim 105-160^{\circ}$. Closer to the phase of the ALMA observations (phase $~ 0.36$ ), the observations of Karovska et al. (1991; at phase 0.43) find a position angle of $\sim 30^{\circ}$. The elongation itself varies with wavelength and phase from none to $\sim 20 \%$, which is consistent with our observations. The wavelength dependence of the elongation is likely to be opacity effects. The apparent variation in position angle with stellar phase could indicate that stable non-radial pulsations shape the extended atmosphere.

The diameter of Mira A also varies with phase by about $10 \%$ and was measured to be $\sim 32$ mas at $2 \mu \mathrm{m}$ wavelength (Woodruff et al. 2004). This corresponds to a Rosseland radius of $\sim 1.5 \mathrm{AU}$. The radius derived from the major axis we measured in Band 3 would thus correspond to $\sim 1.3 R_{*}$ while it corresponds to $\sim 1.35 R_{*}$ in Band 6. Previous observations at $43 \mathrm{GHz}$ resolved the radio photosphere of Mira A and derived a uniform disk diameter of $\sim 52$ mas, corresponding to $\sim 1.6 R_{*}$ Reid \& Menten (2007). That the Band 6 observations appear to probe a larger radius than the Band 3 observations likely requires different opacity sources throughout the atmosphere.

\subsubsection{Emission process}

Reid \& Menten (1997) analyze different centimeter-wavelength continuum emission processes and conclude that the detected emission at $8.5-22 \mathrm{GHz}$ originates in a radio photosphere reaching unity optical depth at around $\sim 2 R_{\star}$, predicting spectral index of $\alpha=1.86$ at centimeter wavelengths. Matthews \& Karovska (2006) published resolved images of the Mira system at $8.5-43.3 \mathrm{GHz}$. The system was monitored with the VLA over $\sim 80 \%$ of the pulsational cycle and showed flux density

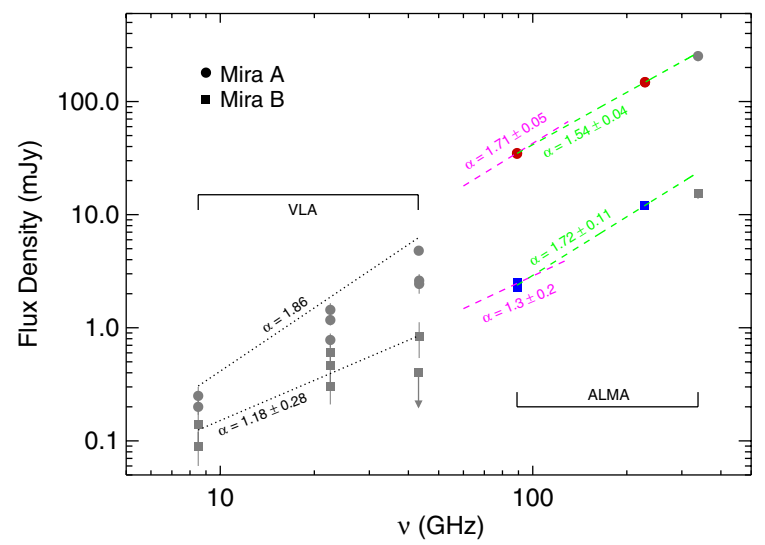

Fig. 3. Mira A and B spectral energy distribution from the radio to the submillimeter frequency range as probed by ALMA and the VLA. The round (red) symbols denote Mira A and the squares (blue) Mira B. The gray symbols are from the literature referred to in the text. The errors on the VLA data points only contain the formal flux density uncertainties, while the ALMA error bars also contain the absolute uncertainties (5\% and 10\% for Bands 3 and 6 together, and Band 7, respectively). The dotted lines indicate the Reid \& Menten (1997) model for Mira A and the fit to the VLA data for Mira B. The dashed line at the Band-3 points is the spectral index derived within the Band, and the green dashed line is the fit to the ALMA Bands 3 and 6 data.

variability $\$ 30 \%$ below $22 \mathrm{GHz}$. The lower frequency data is consistent with the model from Reid \& Menten (1997). At $43 \mathrm{GHz}$ the flux density appears to show larger variability based on the observations by Reid \& Menten (2007) who find a flux density almost a factor of two higher than reported in Matthews \& Karovska (2006). The VLA values, the ALMA values from this work, and the flux density at $345 \mathrm{GHz}$ (Ramstedt et al. 2014) are presented in Fig. 3. The most striking result is that the spectral index between the ALMA Bands 3 and observations $(\alpha=1.54 \pm 0.04)$ appears significantly more shallow than within Band 3 itself $(\alpha=1.70 \pm 0.05)$. The shallower slope agrees with the observations in ALMA Band 7 though the steeper slope would be required to fit the radio observations. Both spectral indexes do not quite agree with the model prediction by Reid \& Menten (1997), indicating that the sources of opacity change at millimeter wavelengths. Our observations yield significantly higher brightness temperature than expected from the VLA observations. For a uniform disk we find $T_{b} \sim 5300 \mathrm{~K}$ at Band $3\left(\sim 1.3 R_{*}\right)$ and $T_{b} \sim 2500 \mathrm{~K}$ at Band 6 $\left(\sim 1.35 R_{*}\right)$, compared to the $T_{b} \sim 1650 \mathrm{~K}$ at $43 \mathrm{GHz}\left(\sim 1.6 R_{*}\right)$. Only part of this difference could be explained by variability, and especially the higher temperatures in Band 3 might require shock heating of the atmosphere close to the stellar surface.

\subsubsection{Stellar activity}

As shown in Fig. 1, the Band 6 data require the presence of a strong compact component with a flux density of $\sim 10 \mathrm{mJy}$ offset by $\sim 3$ mas from the stellar disk center. A Gaussian component of $\sim 4.7$ mas $(\sim 0.4 \mathrm{AU})$ produces a good fit, though we cannot preclude a cluster of delta functions. We have thus detected the presence of a significant hotspot, or a compact cluster of spots, on the Mira A stellar disk at $\sim 1.3 \mathrm{~mm}$ wavelength. We investigated whether the same spot could be detected in the Band 3 observations at $\sim 3 \mathrm{~mm}$. Fits including a Gaussian component up to $\sim 2.5 \mathrm{mJy}$ produced equally good results, but the worse angular resolution did not allow us to confidently distinguish between a fit of a stellar disk with a compact component or a stellar disk alone. 
The flux density measurements allow us to determine the brightness temperature. With a size of $\sim 4.7$ mas, we find $T_{b} \sim$ $10000 \mathrm{~K}$, which is above the brightness temperature of $\sim 2500 \mathrm{~K}$ measured for the stellar disk in Band 6. Assuming a 4.7 mas area, the upper limit in Band 3 gives $T_{b}<17500 \mathrm{~K}$. Bright hotspots at millimeter wavelengths could be caused by shock heating due to pulsations or convection. However, the high brightness temperature of the hotspots at $\sim 1.3 R_{*}$ is more readily explained by magnetic activity as seen on our Sun. Similar magnetic flares were also suggested as the cause of the soft X-ray outburst observed on Mira A in December 2003 (Karovska et al. 2005), but this is the first direct detection of such magnetic activity by an AGB star in the long wavelength regime. The observed hotspot could be related to the strongly polarized elongated $\mathrm{SiO}$ maser ejections that appear aligned with a radial magnetic field (Cotton et al. 2006). Karovska et al. (2005) crudely estimated the lifetime of such magnetic flares to be several weeks. We notice a significant difference of almost $15 \%$ within two days. This would be consistent with the lifetime estimate of a few weeks, but could also indicate significant short term variability.

\subsection{Mira B}

The accreting companion Mira $\mathrm{B}$ has a time-varying accretion-driven wind $\left(\dot{M} \sim 5 \times 10^{-13}-10^{-11} M_{\odot} \mathrm{yr}^{-1}, v_{\infty} \sim\right.$ 250-450 $\mathrm{km} \mathrm{s}^{-1}$, Wood \& Karovska 2006), and recently ALMA has shown the impact of this fast and tenuous wind on the much slower and denser outflow $\left(\dot{M} \sim 10^{-7} M_{\odot} \mathrm{yr}^{-1}, v_{\infty} \sim 10 \mathrm{~km} \mathrm{~s}^{-1}\right)$ of Mira A (Ramstedt et al. 2014). Even if fully ionized, the expected flux density of Mira B's wind would be at least four orders of magnitude lower than what we observe at 89 and $229 \mathrm{GHz}$, and would also be optically thin (i.e., $S_{v} \propto v^{-0.1}$ ). We therefore rule out any significant contribution from Mira B's wind at ALMA frequencies. The steep spectral index of Mira B ( $\alpha \sim 1.3-1.7)$ also rules out any significant non-thermal component, while the symmetric nature of the resolved emission makes it unlikely that the emission is being produced from a collision of the two winds. Our derived diameter for Mira B is $2.4 \mathrm{AU}$ which is two orders of magnitude greater than the size of the accretion disk detected by Reimers \& Cassatella (1985), so we do not directly detect the accretion disk at ALMA frequencies.

Matthews \& Karovska (2006) treated the centimeter emission from Mira B so that it would emanate from an ionized cavity in Mira A's wind, formed by the UV radiation field of the hot accretion disk. They approximated a diameter of 1-5 AU for Mira B, which is consistent with our findings. However, following Taylor \& Seaquist (1984) and assuming a gas temperature of $T=10^{4} \mathrm{~K}$, the spectral energy distribution for such an ionized cavity should have turned over to a spectral index of $\alpha=-0.1$, so well below our ALMA frequencies. Instead we find that $\alpha=1.3 \pm 0.2$ at $89 \mathrm{GHz}$, which is consistent with the $\alpha=1.18 \pm 0.28$ value of Matthews \& Karovska (2006) at lower frequencies. The absence of a turnover in the spectral index up to $89 \mathrm{GHz}$ implies that the gas density at the location of Mira B is at the very least an order of magnitude greater than the gas density of Mira A's wind at that location. It is therefore likely that we are detecting a gravitationally bound, partially ionized gas at centimeter and (sub-)millimeter wavelengths and not an ionized cavity of Mira A's wind. We also find that the spectral index steepens to $\alpha=1.72 \pm 0.11$ between 89 and $229 \mathrm{GHz}$, which could be due to additional dust emission at a few tens of $\mathrm{K}$.

\section{Conclusions}

With the longest ALMA baselines, we have, for the first time, been able to resolve both the extended atmosphere of Mira A and the partially ionized region around Mira B at millimeter wavelengths. The partially ionized region of $\sim 2.4$ AU around Mira B requires a density at least an order of magnitude higher than expected from the Mira A wind. This supports the suggestion that the region is made up of gravitationally captured material from the AGB wind that will be accreted onto Mira B through its accretion disk. The elongation and wavelength-dependent size of Mira A point to strong changes in the opacity source throughout the extended envelope and possible non-radial pulsations. The brightness temperature at $3 \mathrm{~mm}$ is significantly higher than predicted in the radio photosphere model from Reid \& Menten (1997). At $1.3 \mathrm{~mm}$ wavelength we also find a hotspot with an area of $\sim 8 \%$ of the stellar disk. The hotspot has $T_{b} \sim 10000 \mathrm{~K}$. This indicates an origin most likely connected to magnetic activity and potentially related to the process responsible for the previously observed X-ray outburst and other recorded transient phenomena. The stellar activity could also explain the hot layers of the atmosphere probed by the $3 \mathrm{~mm}$ observations.

Acknowledgements. This paper makes use of the following ALMA data: ADS/JAO.ALMA\#2011.0.00014.SV. ALMA is a partnership of ESO (representing its member states), NSF (USA), and NINS (Japan), together with NRC (Canada), NSC and ASIAA (Taiwan), and KASI (Republic of Korea), in cooperation with the Republic of Chile. The Joint ALMA Observatory is operated by ESO, AUI/NRAO, and NAOJ. This research is supported by Marie Curie Career Integration Grant 321691, the Swedish Research Council (VR), and the European Research Council through ERC consolidator grant 614264.

\section{References}

ALMA partnership, Fomalont, E., Vlahakis, C., et al. 2015, ApJ, in press [arXiv: 1504.04877]

Cotton, W. D., Vlemmings, W., Mennesson, B., et al. 2006, A\&A, 456, 339

Etoka, S., Gérard, E., Richards, A., et al. 2010, in 10th European VLBI Network Symp. and EVN Users Meeting: VLBI and the New Generation of Radio Arrays, 6

Haniff, C. A., Ghez, A. M., Gorham, P. W., et al. 1992, AJ, 103, 1662

Karovska, M., Nisenson, P., Papaliolios, C., \& Boyle, R. P. 1991, ApJ, 374, L51 Karovska, M., Hack, W., Raymond, J., \& Guinan, E. 1997, ApJ, 482, L175

Karovska, M., Schlegel, E., Hack, W., Raymond, J. C., \& Wood, B. E. 2005, ApJ, 623, L137

Martí-Vidal, I., Vlemmings, W. H. T., Muller, S., \& Casey, S. 2014, A\&A, 563, A136

Matthews, L. D., \& Karovska, M. 2006, ApJ, 637, L49

Prieur, J. L., Aristidi, E., Lopez, B., et al. 2002, ApJS, 139, 249

Quirrenbach, A., Mozurkewich, D., Armstrong, J. T., et al. 1992, A\&A, 259, L19

Ramstedt, S., Mohamed, S., Vlemmings, W. H. T., et al. 2014, A\&A, 570, L14

Reid, M. J., \& Menten, K. M. 1997, ApJ, 476, 327

Reid, M. J., \& Menten, K. M. 2007, ApJ, 671, 2068

Reimers, D., \& Cassatella, A. 1985, ApJ, 297, 275

Taylor, A. R., \& Seaquist, E. R. 1984, ApJ, 286, 263

van Leeuwen, F. 2007, A\&A, 474, 653

Wilson, R. W., Baldwin, J. E., Buscher, D. F., \& Warner, P. J. 1992, MNRAS, 257,369

Wood, B. E., \& Karovska, M. 2006, ApJ, 649, 410

Woodruff, H. C., Eberhardt, M., Driebe, T., et al. 2004, A\&A, 421, 703

Page 5 is available in the electronic edition of the journal at http: //www . aanda. org 


\section{Appendix A: Flux density calibrators}

Several quasars were used for flux density calibration at the different epochs and bands. In Table A.1 we present the calibrators and their adopted flux densities and spectral indices from the ALMA calibrator catalog.

Table A.1. Calibrator fluxes.

\begin{tabular}{lcccc}
\hline \hline Epoch & Calibrator & $\begin{array}{c}\text { Reference } \\
\text { freq. [GHz] }\end{array}$ & $\begin{array}{c}\text { Flux } \\
\text { density [Jy] }\end{array}$ & $\begin{array}{c}\text { Spectral } \\
\text { index }\end{array}$ \\
\hline 17 Oct. 2014 & J0334-4008 & 86.23 & 1.66 & -0.71 \\
25 Oct. 2014 & J2258-2758 & 86.23 & 1.21 & -0.73 \\
29 Oct. 2014 & J0334-4008 & 229.55 & 0.83 & -0.70 \\
01 Nov. 2014 & J0238+1636 & 229.55 & 1.39 & -0.20 \\
\hline
\end{tabular}

\section{Appendix B: Relative astrometry}

The binary pair Mira AB is fully resolved in the observations, with a representative image at the two frequencies presented in Fig. 1 (left) and Fig. 2 (left). Using $u v$-fitting we determine the separation and position angle with milliarcsecond accuracy as indicated in Table B.1. The average separation of $0.472^{\prime \prime}$ corresponds to $43.4 \mathrm{AU}$ at a distance of $92 \mathrm{pc}$. These values fit well with the predictions made by Prieur et al. (2002), although the authors note that their derived binary orbit is still poorly constrained. It is interesting to note that the data already show a (not yet significant) sign of a decrease in separation $R$ over two weeks of observing at a rate of $\sim 121 \pm 71 \mu$ as day ${ }^{-1}$. Futher epochs of observations with ALMA will thus be able to constrain the binary orbit to high precision.

Table B.1. Relative astrometry.

\begin{tabular}{lcc}
\hline \hline Epoch & $\begin{array}{c}\text { Separation } \\
{\left[{ }^{\prime}\right]}\end{array}$ & $\begin{array}{c}\text { Position angle } \\
{\left[{ }^{\circ}\right]}\end{array}$ \\
\hline 17 Oct. 2014 & $0.4722 \pm 0.0005$ & $98.79 \pm 0.07$ \\
25 Oct. 2014 & $0.4721 \pm 0.0005$ & $98.59 \pm 0.06$ \\
29 Oct. 2014 & $0.4719 \pm 0.0002$ & $98.64 \pm 0.02$ \\
01 Nov. 2014 & $0.4709 \pm 0.0001$ & $98.53 \pm 0.02$ \\
\hline
\end{tabular}

